\title{
Poland Syndrome
}

National Cancer Institute

\section{Source}

National Cancer Institute. Poland Syndrome. NCI Thesaurus. Code C85017.

A rare developmental disorder characterized by underdevelopment or absence of the pectoralis muscle in one side of the chest, usually associated with ipsilateral cutaneous syndactyly, and ipsilateral breast and nipple hypoplasia. 